\title{
Manifestaciones pulmonares relacionadas al uso del cigarro electrónico: una revisión de la literatura
}

\section{Pulmonary manifestations related to electronic cigarette use: a literature review}

\author{
Andrea Malagón-Liceaga, Martín Roberto Basile-Álvarez, ${ }^{*}$ Yamil Shahin-Sabines, ${ }^{*}$ José Javier Elizalde-González ${ }^{\ddagger}$
}

*Facultad de Medicina, Universidad Nacional Autónoma de México, Ciudad de México, México.

‡ Instituto Nacional de Ciencias Médicas y Nutrición Salvador Zubirán, Ciudad de México, México.

RESUMEN. El uso del cigarrillo electrónico es cada vez más popular entre los adolescentes y adultos jóvenes mexicanos. La lesión pulmonar asociada al uso de este dispositivo es bien conocida y ya ha causado la muerte de un adulto joven en nuestro país, asimismo, en Estados Unidos de América ha llegado al nivel de epidemia con miles de afectados y decenas de muertes. Aunque el uso del cigarrillo electrónico es cada vez más frecuente en México, hace falta trabajar en el reconocimiento de la lesión pulmonar asociada y su debido reporte epidemiológico. La fisiopatología de esta enfermedad no se ha esclarecido debido a los numerosos compuestos que se añaden a los cigarrillos, pero particularmente relevantes son los diluyentes como el acetato de vitamina E que se añade al tetrahidrocannabinol. El diagnóstico de esta entidad es principalmente de exclusión en pacientes con historia de consumo de estos productos y signos y síntomas de lesión pulmonar. El tratamiento es esencialmente de soporte y considerando infecciones pulmonares de mayor prevalencia. Los profesionales de la salud deben insistir en la suspensión del consumo del cigarrillo electrónico.

Palabras clave: EVALI, cigarrillo electrónico, lesión pulmonar, neumonía, tetrahidrocannabinol.

\section{INTRODUCCIÓN}

La popularidad del cigarrillo electrónico ha aumentado en años recientes. Se trata de un dispositivo electrónico activado con una batería, el cual produce aerosoles (o vapores, lo que da a su uso el nombre de vaping en inglés) que con-

Correspondencia:

Dr. José Javier Elizalde-González

Instituto Nacional de Ciencias Médicas y Nutrición «Salvador

Zubirán», Ciudad de México.

Correo electrónico: javier.elizaldeg@incmnsz.mx

Recibido: 24-II-2021; aceptado: 07-IV-2021.

Citar como: Malagón-Liceaga A, Basile-Álvarez MR, Shahin-Sabines Y, Elizalde-González JJ. Manifestaciones pulmonares relacionadas al uso del cigarro electrónico: una revisión de la literatura. Neumol Cir Torax. 2021; 80 (3): 197-203. https://dx.doi.org/10.35366/102480
ABSTRACT. The use of electronic cigarettes is becoming increasingly popular among Mexican adolescents and young adults. The lung injury associated with the use of this device is well recognized and has already caused the death of a young adult in our country, likewise, in the United States it has reached an epidemic level with thousands of people affected and dozens of deaths. The pathophysiology of this disease has not been elucidated due to the numerous compounds added to these cigarettes, but diluents such as vitamin E acetate added to tetrahydrocannabinol are particularly relevant. The diagnosis of this entity is mainly one of exclusion in patients with a history of use of these products, and signs and symptoms of lung injury. Management is essentially supporting treatment, considering also more prevalent pulmonary infections. Health professionals should insist on the suspension of electronic cigarette use.

Keywords: EVALI, electronic cigarette, lung injury, pneumonia, tetrahydrocannabinol.

tiene, además de nicotina, saborizantes y otros químicos como glicerina y propilenglicol; se ha utilizado también para el consumo de tetrahidrocannabinol $(\mathrm{THC})^{1-3}$ y se estima que ha aumentado en más de 10\% el tabaquismo entre los adolescentes norteamericanos. Estos dispositivos se inventaron en China en 2003 y a partir de entonces se han desarrollado varias generaciones del mismo; ${ }^{4}$ en la actualidad, se encuentran en circulación los cigarrillos electrónicos de cuarta generación. Desde 2012 comenzaron a salir los primeros reportes sobre la asociación entre la práctica denominada vaping y el desarrollo de lesión pulmonar, ${ }^{4}$ llegando eventualmente al nivel de brote nacional entre adultos jóvenes en Estados Unidos en 2019.' La enfermedad fue denominada como EVALI (por sus siglas en inglés, Ecigarette or Vaping Product Use-Associated Lung Injury). ${ }^{3}$ El uso de estos dispositivos se ha generalizado mundialmente, incluyendo a México, principalmente entre los jóvenes a pesar de las prohibiciones para su importación en nuestro 
país. ${ }^{5}$ En relación con lo anterior, enfocamos el presente texto en la revisión de su epidemiología, fisiopatología, diagnóstico y tratamiento.

\section{Epidemiología y factores de riesgo}

Se desconocen datos precisos acerca de la incidencia y prevalencia de esta enfermedad. Se calcula que, en México, $10 \%$ de los adolescentes han probado el cigarrillo electrónico, $4 \%$ lo usan de forma exclusiva ${ }^{5}$ y se ha reportado una muerte por EVALI en el país. Dada la distribución por edades y el rápido aumento en su uso, no es sorprendente que los informes de EVALI se encuentren principalmente entre adolescentes y adultos jóvenes. ${ }^{3}$ En 2019 hubo múltiples brotes en Estados Unidos de América que llegaron a considerarse una epidemia, con más de 2,000 casos y más de 60 fallecidos a inicios del año 2020. ${ }^{3}$ En esta misma epidemia, una caracterización de los pacientes identificó que los afectados eran predominantemente adultos y adolescentes menores de 35 años, blancos y varones, además, se informó el uso de productos con THC en alrededor de $85 \%$ de los pacientes. ${ }^{6}$

El uso de cigarrillos electrónicos constituye un factor de riesgo indispensable para desarrollar la enfermedad; sus productos pueden contener múltiples componentes asociados con EVALI, particularmente el propilenglicol, el acetato de vitamina $\mathrm{E}$ y metales como el plomo y el arsénico. ${ }^{3}$ En Estados Unidos se identificó al acetato de vitamina $\mathrm{E}$ en el líquido del lavado broncoalveolar (BAL, por sus siglas en inglés) en más de $90 \%$ de los pacientes estudiados y en más del 90\% se confirmó el uso de THC, proponiendo que la dilución del THC con acetato de vitamina $\mathrm{E}$ en el mercado ilegal fue responsable en gran medida de la epidemia. ${ }^{7}$ Por esto, se ha considerado un factor de riesgo adquirir los cigarrillos electrónicos de manera ilícita, así como el uso de ciertas prácticas como el dabbing, que se refiere a la inhalación de vapores concentrados de las sustancias para aumentar sus efectos. ${ }^{3}$ El Departamento de Salud Pública de Illinois realizó una encuesta en línea que asoció significativamente los factores de riesgo asociados al desarrollo de EVALI durante el brote nacional de 2019 en Estados Unidos de América, los cuales son: ${ }^{8}$

1. Uso exclusivo de productos de vaping para el consumo de THC.

2. Uso frecuente (más de cinco veces al día) de THC.

3. Obtener los productos de consumo de fuentes ilegales.

\section{Fisiopatología}

Hasta el momento se desconoce el mecanismo fisiopatológico exacto de la enfermedad, y la heterogeneidad de los productos en los cigarrillos electrónicos dificulta su esclarecimiento. ${ }^{9}$ Las diferentes presentaciones clínicas probablemente se deban a enfermedades subyacentes, variaciones individuales en la respuesta a sustancias inhaladas, además del mismo material inhalado. ${ }^{3}$ Los cigarrillos contienen múltiples grupos de compuestos potencialmente tóxicos: nicotina, carbonilos, compuestos orgánicos volátiles (como benceno y tolueno), partículas y trazas de metales, endotoxinas bacterianas y elementos de algunos hongos. ${ }^{10}$ Histológicamente, las biopsias pulmonares muestran características de lesión pulmonar aguda en cualquiera de sus fases (edema, formación de membranas hialinas, depósito de fibrina, infiltrado de células inflamatorias y formación de tejido fibroblástico). ${ }^{11}$ Entre los patrones específicos de lesión pulmonar se han descrito: neumonía lipoidea, neumonía aguda eosinofílica, neumonitis por hipersensibilidad, hemorragia alveolar difusa, bronquiolitis respiratoria-enfermedad intersticial pulmonar, neumonía organizada, neumonitis granulomatosa y neumonitis intersticial de células gigantes. ${ }^{9}$ Un hallazgo constante es la presencia de macrófagos intraalveolares, caracterizados por tener un citoplasma finamente vacuolado compatible con neumonía lipoidea endógena (fagocitosis de material lipídico celular por lesión epitelial); si bien, estos hallazgos no son específicos, son característicos de neumonitis inducida por químicos.10,11 El infiltrado neutrofílico es generalmente prominente y los eosinófilos son más raros. ${ }^{12}$ Estos hallazgos apoyan la teoría de que no hay un único mecanismo de daño.

Entre los agentes responsables de la toxicidad pulmonar, algunos diluyentes de productos con nicotina, como el propilenglicol y el glicerol, han demostrado en modelos animales causar daño relacionado con el deterioro de la homeostasis de los lípidos y de la respuesta inmune del huésped, ${ }^{3}$ de igual forma al ser expuestos a altas temperaturas, se descomponen para producir formaldehídos, acroleína y acetaldehído que resultan tóxicos para el epitelio respiratorio. ${ }^{9}$ Algunos saborizantes, como los aldehídos (diacetil y acetil propionil), han causado bronquiolitis al ser inhalados durante el mismo proceso de manufactura ${ }^{9}$ y tanto el diacetil como el 2,3-pentanodiol interrumpen las vías de expresión génica relacionadas con los cilios respiratorios y el citoesqueleto de las células epiteliales bronquiales. ${ }^{10}$ Un estudio detectó precisamente diacetil en varias marcas de cigarrillos electrónicos, en sabores como sandía, melocotón y granada. ${ }^{13} \mathrm{El}$ acetato de vitamina $\mathrm{E}$, utilizado como diluyente para el THC, produce al calentarse un compuesto denominado cetena que actúa como irritante pulmonar, promoviendo la activación de la compleja cascada inflamatoria. ${ }^{3,9}$ Cuando se inhala, el mismo acetato de vitamina $\mathrm{E}$ se incorpora a los fosfolípidos que componen el surfactante pulmonar, aumentando su permeabilidad y la tensión superficial de los alvéolos. ${ }^{13}$ Se sabe además que su descomposición térmica puede generar compuestos secundarios potencialmente dañinos..$^{13}$ 
Existe cada vez más evidencia de que la toxicidad de estos productos no se limita únicamente a los diluyentes y saborizantes. Hay estudios in vitro que demostraron una disminución en la viabilidad de las células epiteliales bronquiales humanas después de su exposición al vapor de los cigarrillos electrónicos, probablemente debido al daño al ADN, agotamiento de las reservas de glutatión y aumento de la permeabilidad de la membrana celular. ${ }^{13} \mathrm{Se}$ ha encontrado en modelos murinos un aumento de ciertas citocinas proinflamatorias (como IL-6, MCP-1, IL-1 $\alpha$ e IL-13) y se ha visto que a medida que la bobina del cigarrillo sufre ciclos repetidos de calentamiento, las moléculas de metales traza pueden alcanzar el líquido y posteriormente al epitelio pulmonar, encontrando evidencia de restos de aluminio, calcio, cromo, cobre, moléculas de hierro, plomo, magnesio, estaño y zinc. ${ }^{13}$ Éstos son algunos de los mecanismos patogénicos dilucidados, aunque se debe enfatizar que aún hay muchos que faltan por conocer.

\section{Diagnóstico}

Definiciones: hasta la fecha no se han consensado criterios diagnósticos formales para EVALI. Las presentaciones heterogéneas sugieren que EVALI comprende varias formas diferentes de lesión pulmonar, y su diagnóstico debe basarse principalmente en la exclusión de infecciones pulmonares o algún otro diagnóstico alternativo probable (p. ej. de origen cardíaco, reumatológico, entre otras). ${ }^{9}$ Los criterios diagnósticos sugestivos actuales para EVALI se detallan a continuación:

1. Síntomas sistémicos inespecíficos (p. ej. fiebre, escalofríos y taquicardia) y síntomas respiratorios (p. ej. tos, disnea, hipoxemia, dolor torácico).

2. Uso de cigarrillos electrónicos (o productos relacionados) 90 días antes del inicio de los síntomas.

3. Detección de opacidades pulmonares en estudios de imagen.

4. Ausencia de otras causas probables (p. ej. infección, presencia de neoplasias, cardíaco).

Manifestaciones clínicas: cuando se sospecha de una lesión pulmonar por el uso de cigarrillos electrónicos, como se menciona en definiciones, no se conocen signos ni síntomas patognomónicos. ${ }^{14}$ Se debe obtener una historia clínica completa, esta debe incluir: cuándo se comenzó el uso del cigarro electrónico, frecuencia de consumo con énfasis en los últimos 90 días, qué dispositivo se utiliza, así como el sabor y marca del líquido que usa, se debe preguntar de igual forma si éste es usado para el consumo de THC. ${ }^{15}$ Se debe sospechar de EVALI en pacientes que cuenten con el antecedente de uso de estos productos y que refieren sintomatología respiratoria, gastrointestinal o constitucional..$^{12}$ Por la relativa baja frecuencia y carencia de una prueba diagnóstica definitiva, se maneja como un diagnóstico de exclusión, el cual es apoyado por imagen de tórax consistente con EVALI. Los síntomas característicos, al ser inespecíficos, deben investigarse a fondo, pues éstos se presentan en muchas entidades diferentes siendo la gran mayoría de origen infeccioso. ${ }^{12}$ Estos son: tos, disnea, dolor torácico, fiebre, pérdida de peso, dolor abdominal, diarrea y náusea o vómito; la exploración física debe estar enfocada a datos sutiles de fiebre, taquicardia e hipoxemia y a una monitorización constante de la oximetría de pulso y la frecuencia respiratoria. ${ }^{12}$

Laboratorio: como ya se mencionó, no existe una prueba diagnóstica definitiva. Cuando se sospecha de EVALI existen ciertos hallazgos que aumentan la probabilidad del diagnóstico y, a su vez, sirven para descartar una infección. Se indica una biometría hemática en la cual es frecuente encontrar una leucocitosis con marcada neutrofilia, elevación de transaminasas hepáticas, así como datos de inflamación que se detectan con los valores de proteína C reactiva ultrasensible y sedimentación eritrocítica, los niveles altos de procalcitonina sugieren la presencia de infección bacteriana. ${ }^{12}$ Otra prueba de laboratorio que se utiliza es la prueba toxicológica de THC en orina ${ }^{15} \mathrm{O}$ una prueba en orina para detectar el antígeno del Streptococcus pneumoniae como parte del diagnóstico diferencial.

Gabinete: cuando se recurre a una broncoscopia con la realización de $\mathrm{BAL}$, se ha encontrado predominante neutrofilia, ${ }^{12}$ sin embargo, esta prueba es especializada y no está indicada de rutina. En reportes de casos de EVALI, se encontró eosinofilia de hasta $26 \%$ con cultivos para hongos, virus y bacterias negativos, así descartando causas de origen infeccioso, por esto, aunque no es el patrón más frecuente, ${ }^{16}$ se concluye que existe evidencia de una neumonía eosinofílica aguda secundaria al uso del cigarrillo electrónico.

Imagen: ante la sospecha de EVALI o síntomas inespecíficos en pacientes con consumo recurrente de cigarrillos electrónicos, se indica una telerradiografía de tórax. El patrón comúnmente detectado en estos pacientes es una atenuación en vidrio esmerilado difuso bilateral con predominio basal, éste respeta la región subpleural. ${ }^{15,17}$ En menor frecuencia se han detectado otros patrones, entre ellos, imágenes compatibles con daño alveolar difuso, neumonía lipoidea y neumonía organizada. También existe evidencia de neumotórax, neumomediastino y derrame pleural. ${ }^{17}$

Se puede recurrir a una tomografía axial computarizada, particularmente si no se detecta nada en la radiografía. ${ }^{17}$ Estos hallazgos se pueden encontrar en muchas otras alteraciones, lo que conlleva una extensa lista de diagnósticos diferenciales, en ésta se destacan: neumonía bacteriana atípica, hemorragia pulmonar, daño por inhalantes, reacción secundaria a drogas, neumonitis aguda por hipersensibilidad, neumonía intersticial, etcétera. ${ }^{9,17}$ 
En el Apéndice 1 se muestra el algoritmo diagnóstico para EVALI.

\section{Tratamiento}

En la actualidad no existe un consenso para el tratamiento estandarizado de pacientes con EVALI. Si bien es cierto que existe una gran cantidad de diagnósticos diferenciales que deben excluirse antes de realizar formalmente el diagnóstico, el pilar fundamental al momento de comenzar el tratamiento es garantizar que la neumonía adquirida en la comunidad (NAC) no sea tratada de forma insuficiente o incluso llegar a ser pasada por alto, especialmente considerando que su prevalencia es mucho más común. Por lo anterior, la utilización de antibióticos empíricos basados en las guías locales para NAC está fuertemente recomendada, especialmente cuando se encuentran pendientes los resultados de las evaluaciones iniciales de la enfermedad. ${ }^{17,18}$

Se debe considerar también la interconsulta con un neumólogo, quien puede ayudar a guiar una evaluación adicional y diagnóstico diferencial, recomendar el uso de antibióticos empíricos, manejo avanzado de la insuficiencia respiratoria y revisar las indicaciones de broncoscopia en cada paciente en lo particular. En caso de que no haya evidencia de infección pulmonar o en torrente sanguíneo posterior a la iniciación de antibióticos empíricos para NAC, se puede realizar una disminución secuencial en la dosis de antibióticos. ${ }^{9}$ Dentro de los pacientes que se presentan con EVALI, se ha visto que más de 95\% requieren hospitalización. ${ }^{19}$ Los criterios más importantes para admisión hospitalaria son: aquellos pacientes con posible lesión pulmonar, especialmente si hay dificultad respiratoria, que además puedan tener comorbilidades que comprometan la reserva pulmonar o que presenten una saturación menor al 95\% a nivel del mar. La mayoría de los pacientes requieren apoyo con oxígeno, buscando mantener una saturación de oxígeno entre 88 y 92\%; no obstante, dependiendo de la gravedad de la presentación, se puede optar por la utilización de terapia con oxígeno de alto flujo o ventilación no invasiva con CPAP (continuous positive airway pressure) para reducir el trabajo respiratorio. En caso de que la hipoxemia empeore, el tratamiento es similar al del síndrome de insuficiencia respiratoria progresiva aguda (SIRPA). El uso de ventilación mecánica a lo largo de distintas series ha variado, pero oscila entre 26 y $35 \% \%^{9,14}$ Los pacientes ambulatorios deben tener niveles normales de saturación de oxígeno, ausencia de datos de dificultad respiratoria y una buena base de apoyo familiar, así como acceso confiable a los servicios de atención médica y deben ser instruidos para buscar atención médica oportuna si los síntomas respiratorios empeoran. ${ }^{9}$

La administración de corticoides en pacientes con EVALI requiere de una evaluación individualizada y cuidadosa por parte del especialista. Si bien los corticoides se han utilizado ampliamente en el tratamiento de la lesión pulmonar asociada con cigarrillos electrónicos, su eficacia no ha sido bien estudiada. ${ }^{14,20}$ A pesar de que la administración de corticoides no cuenta con indicaciones específicas, usualmente se reserva su uso en pacientes que cumplen con criterios de EVALI y que además demuestran síntomas de empeoramiento progresivo e hipoxemia. Por otro lado, es razonable suspender el inicio de glucocorticoides en aquellos pacientes con presentaciones menos graves o mientras se evalúa a los pacientes en búsqueda de etiologías infecciosas (p. ej. neumonía fúngica, la cual puede empeorar con el tratamiento con glucocorticoides). ${ }^{17}$ En caso de optarse por la utilización de glucocorticoides, la mayoría de los pacientes en distintas series han mostrado una excelente respuesta con metilprednisolona en un rango de $0.5-1 \mathrm{mg} / \mathrm{kg}$ disminuyendo en cinco a 10 días y reevaluando su curso clínico. ${ }^{14,20}$

Aquellos pacientes que recibieron tratamiento hospitalario por EVALI y posteriormente fueron dados de alta deben tener una visita de seguimiento en un período de tiempo no mayor a una o dos semanas. Debido a los problemas de recurrencia de la enfermedad es necesario asegurarse de que los signos vitales, los niveles de oxigenación y la tolerancia al ejercicio sean estables durante 24 a 48 horas antes del alta después de la hospitalización por EVALI, ${ }^{21}$ pudiéndose efectuar una prueba de caminata de seis minutos.

El seguimiento debe incluir la evaluación del curso clínico del paciente, oximetría de pulso y considerar la realización de al menos una telerradiografía de tórax. Otras pruebas de seguimiento adicionales que se deben realizar de uno a dos meses después del alta incluyen la espirometría, pruebas de capacidad de difusión y otra telerradiografía de tórax. Algunos pacientes han tenido hipoxemia persistente (saturación de oxígeno $<95 \%$ ), requiriendo oxígeno domiciliario en el momento del alta y pueden necesitar un seguimiento médico continuo y estrecho con el neumólogo. ${ }^{17}$

Por último, se debe aconsejar a los pacientes que dejen de utilizar cigarrillos electrónicos (o cualquier tipo de vapeo) y enfatizar la importancia del seguimiento ambulatorio para evitar secuelas o complicaciones agudas que puedan poner en peligro la vida de no ser tratadas después de ser dados de alta del hospital. ${ }^{17}$

En el Apéndice 2 se muestra el algoritmo terapéutico para EVALI.

\section{CONCLUSIONES}

El aumento de la popularidad del cigarrillo entre adolescentes y adultos jóvenes mexicanos resalta la importancia de que los profesionales de la salud aprendan a sospechar, diagnosticar y tratar la enfermedad relacionada a su uso, EVALI. El presente artículo brinda una guía rápida y práctica para este propósito. 


\section{REFERENCIAS}

1. Davidson K, Brancato A, Heetderks P, Mansour W, Matheis E, Nario $\mathrm{M}$, et al. Outbreak of electronic-cigarette- associated acute lipoid pneumonia-North Carolina, july-august 2019. MMWR Morb Mortal Wkly Rep. 2019;68(36):784-786. doi: 10.15585/mmwr.mm6836e1.

2. Khan MS, Khateeb F, Akhtar J, Khan Z, Lal A, Kholodovych V, et al. Organizing pneumonia related to electronic cigarette use: A case report and review of literature. Clin Respir J. 2018;12(3):1295-1299. doi: 10.1111/crj.12775.

3. Belok SH, Parikh R, Bernardo J, Kathuria H. E-cigarette, or vaping, product use- associated lung injury: a review. Pneumonia (Nathan). 2020;12:12. doi: 10.1186/s41479-020-00075-2.

4. Jonas $A M$, Raj $R$. Vaping-related acute parenchymal lung injury: a systematic review. Chest. 2020;158(4):1555-1565. doi: 10.1016/j. chest.2020.03.085.

5. Instituto Nacional De Salud Pública [Internet]. El cigarrillo electrónico, un riesgo para jóvenes mexicanos. [Consultado el 25 de noviembre del 2020]. 2020. Disponible en: https://www.insp.mx/avisos/3789cigarro-electronico-riesgo.html

6. Moritz ED, Zapata LB, Lekiachvili A, Glidden E, Annor FB, Werner AK, et al. Update: characteristics of patients in a national outbreak of E-cigarette, or vaping, product use-associated lung injuries - United States, October 2019. MMWR Morb Mortal Wkly Rep. 2019;68(43):985-989. doi: 10.15585/mmwr.mm6843e1.

7. Blount BC, Karwowski MP, Shields PG, Morel-Espinosa M, ValentinBlasini L, Gardner M, et al. Vitamin E acetate in bronchoalveolarlavage fluid associated with EVALI. N EngI J Med. 2020;382(8):697705. doi: 10.1056/nejmoa1916433.

8. Navon L, Jones CM, Ghinai I, King BA, Briss PA, Hacker KA, et al. Risk factors for E-cigarette, or vaping, product use-associated lung injury (EVALI) among adults who use e-cigarette, or vaping, products-lllinois, July-October 2019. MMWR Morb Mortal Wkly Rep. 2019;68(45):1034-1039. doi: 10.15585/mmwr.mm6845e1.

9. Cherian SV, Kumar A, Estrada-Y-Martin RM. E-cigarette or vaping product- associated lung injury: a review. Am J Med. 2020;133(6):657663. doi: 10.1016/j.amjmed.2020.02.004.

10. Christiani DC. Vaping-induced acute lung injury. N Engl J Med. 2020;382(10):960-962. doi: 10.1056/nejme1912032.

11. Smith ML, Gotway MB, Crotty Alexander LE, Hariri LP. Vaping-related lung injury. Virchows Arch. 2020;1-8. doi: 10.1007/s00428-020-02943-0.

12. Winnicka L, Shenoy MA. EVALI and the pulmonary toxicity of electronic cigarettes: a review. J Gen Intern Med. 2020;35(7):21302135. doi: 10.1007/s11606-020-05813-2.
13. Dicpinigaitis PV, Trachuk P, Fakier F, Teka M, Suhrland MJ. Vapingassociated acute respiratory failure due to acute lipoid pneumonia. Lung. 2020;198(1):31-33. doi: 10.1007/s00408-019-00277-6.

14. Layden JE, Ghinai I, Pray I, Kimball A, Layer M, Tenforde MW, et al. Pulmonary illness related to E-cigarette use in Illinois and Wisconsin-final report. N Engl J Med. 2020;382:903-916. doi: 10.1056/ NEJMoa1911614.

15. Conuel EJ, Chieng HC, Fantauzzi J, Pokhrel K, Goldman C, Smith TC, et al. Cannabinoid oil vaping-associated lung injury and its radiographic appearance. Am J Med. 2020;133(7):865-867. doi: 10.1016/j.amjmed.2019.10.032.

16. Arter ZL, Wiggins A, Hudspath C, Kisling A, Hostler DC, Hostler JM. Acute eosinophilic pneumonia following electronic cigarette use. Respir Med Case Rep. 2019;27:100825. doi: 10.1016/j. rmcr.2019.100825.

17. Jatlaoui TC, Wiltz JL, Kabbani S, Siegel DA, Koppaka R, Montandon $\mathrm{M}$, et al. Update: interim guidance for health care providers for managing patients with suspected E-cigarette, or vaping, product use-associated lung injury-United States, November 2019. MMWR Morb Mortal Wkly Rep. 2019;68(46):1081-1086. doi: 10.15585/mmwr. $\mathrm{mm} 6846 \mathrm{e} 2$.

18. Fonseca Fuentes X, Kashyap R, Hays JT, Chalmers S, Lama von Buchwald C, Gajic O, et al. VpALI-vaping-related acute lung injury: a new killer around the block. Mayo Clin Proc. 2019;94(12):2534-2545. doi: 10.1016/..mayocp.2019.10.010.

19. Chatham-Stephens K, Roguski K, Jang Y, Cho P, Jatlaoui TC, Kabbani S, et al. Characteristics of hospitalized and nonhospitalized patients in a nationwide outbreak of E-cigarette, or vaping, product use-associated lung injury-United States, November 2019. MMWR Morb Mortal Wkly Rep. 2019;68(46):1076-1080. doi: 10.15585/mmwr. mm6846e1.

20. Triantafyllou GA, Tiberio PJ, Zou RH, Lamberty PE, Lynch MJ, Kreit JW, et al. Vaping-associated acute lung injury: a case series. Am J Respir Crit Care Med. 2019;200(11):1430-1431. doi: 10.1164/ rccm.201909-1809le.

21. Mikosz CA, Danielson M, Anderson KN, Pollack LA, Currie DW, Njai $\mathrm{R}$, et al. Characteristics of patients experiencing rehospitalization or death after hospital discharge in a nationwide outbreak of E-cigarette, or vaping, product use-associated lung injury - United States, 2019. MMWR Morb Mortal Wkly Rep. 2020;68(5152):1183-1188. doi: 10.15585/mmwr.mm685152e1.

Conflicto de intereses: Los autores declaran no tener conflicto de intereses. 
Neumol Cir Torax. 2021; 80 (3): 197-203

Apéndice 1: Algoritmo diagnóstico de EVALI (E-cigarette or Vaping Product Use-Associated Lung Injury). $\mathrm{TC}=$ tomografía computarizada; $\mathrm{NAC}=$ neumonía adquirida en la comunidad; $\mathrm{VIH}=$ virus de la inmunodeficiencia humana.

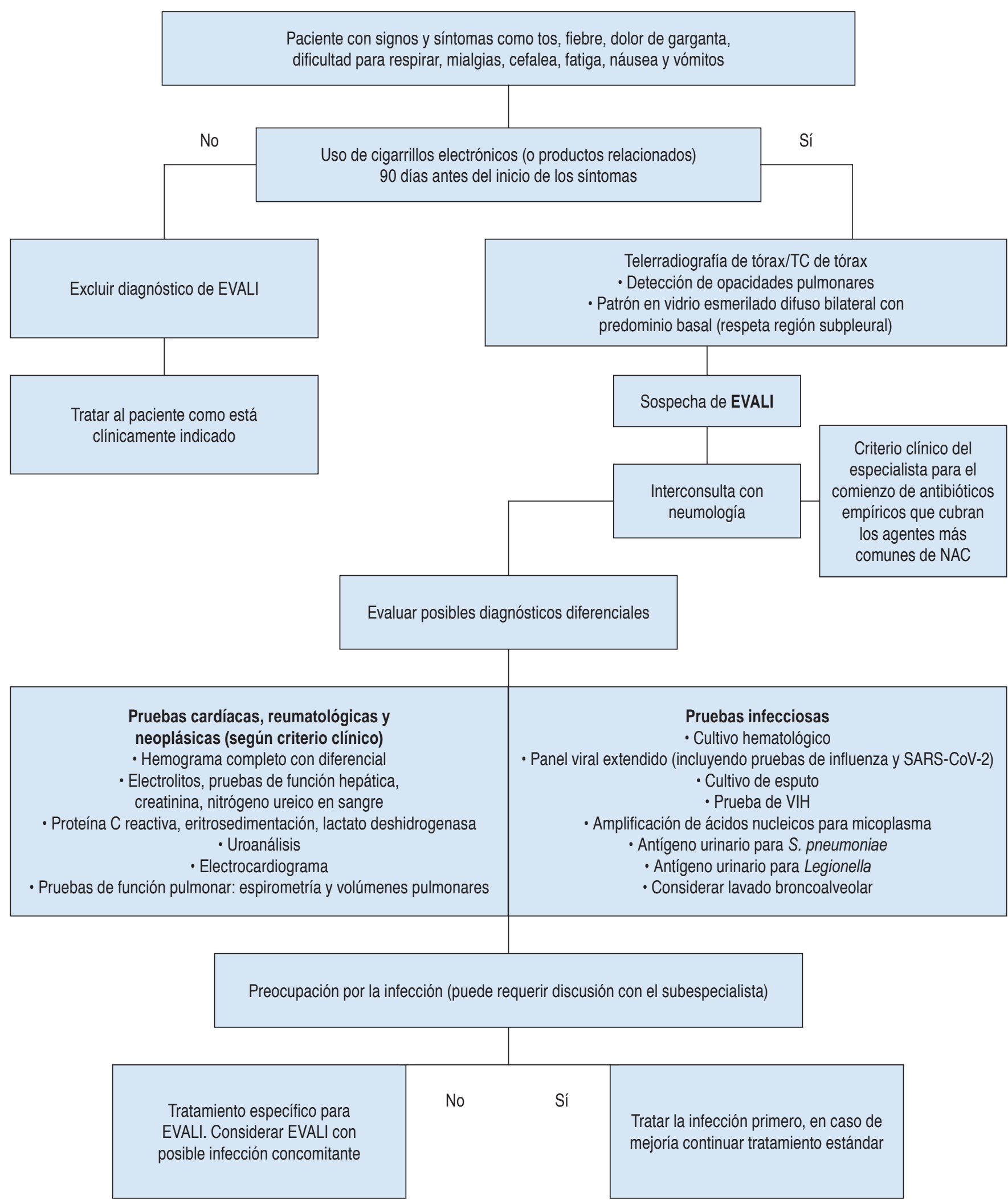


Apéndice 2: Algoritmo terapéutico de EVALI.

$\mathrm{NAC}=$ neumonía adquirida en la comunidad; $\mathrm{CPAP}=$ continuous positive airway pressure.

Si se sospecha de EVALI, el manejo clínico debe proceder de inmediato y ser guiado por los resultados de laboratorio individuales

Criterio clínico del especialista para el comienzo de antibióticos empíricos basados en las guías locales para NAC
En caso de ausencia de infección pulmonar o en torrente sanguíneo posterior a la administración de antibióticos empíricos, realizar una disminución secuencial de la dosis de antibióticos

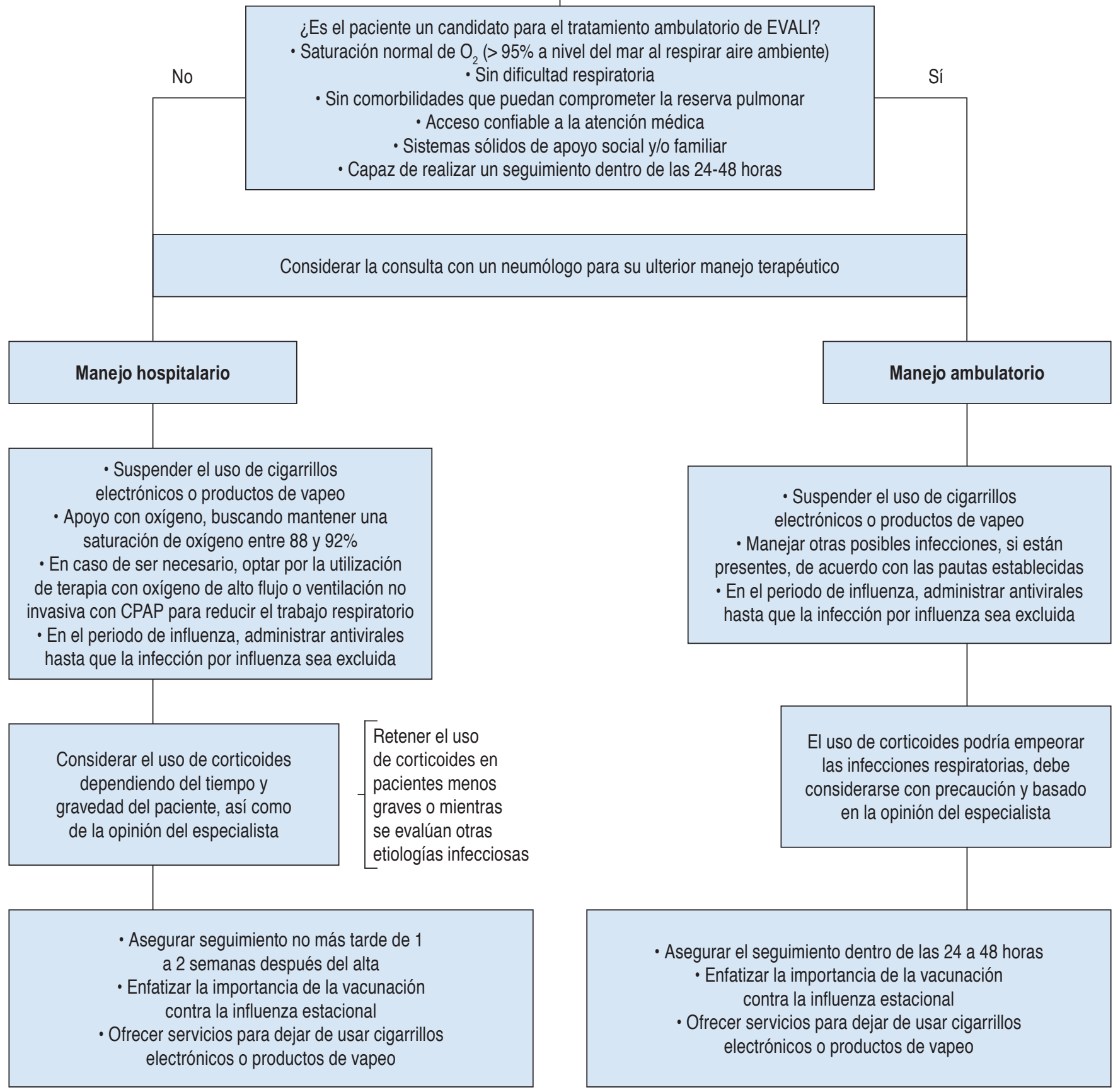

